\title{
Heterogeneous multicore fiber for optical beamforming
}

\author{
Sergi García, Mario Ureña and Ivana Gasulla \\ ITEAM Research Institute, Universitat Politècnica de València, Valencia, Spain \\ sergarc3@iteam.upv.es
}

\begin{abstract}
We experimentally demonstrate, for the first-time to our knowledge, optical beamforming for microwave phased array antennas implemented with a heterogeneous multicore fiber link. The multicore fiber has been engineered to act as an optical sampled true time delay that allows to implement radiofrequency signal processing in a distributed way. It comprises 7 trench-assisted cores where each core is fabricated with different dimensions and core dopant concentration, as to feature a different group delay and chromatic dispersion behavior. We emulated different radio beamsteering scenarios where the beam-pointing angle is modified by tuning the optical wavelength in a 20-nm range, while squint-beam effects are avoided.
\end{abstract}

Keywords-Microwave Photonics, optical beamforming networks, multicore fibers.

\section{INTRODUCTION}

Multicore optical fibers (MCFs) were initially proposed as a compact solution for high-capacity digital communications, [1]. But they exhibit great potential not only to distribute parallel channels but also to process the signal while it is being distributed. This is particularly interesting for next-generation fiber-wireless communications scenarios, as 5G and the Internet of Things, which demand on higher degrees of capacity, connectivity, flexibility and compactness, [2]. In this context, we have previously proposed the exploitation of heterogeneous MCFs as a compact solution for fiber-distributed signal processing, that is, implementing simultaneously both radiofrequency (RF) access distribution (including multiple antenna connectivity) and broadband microwave photonics (MWP) signal processing applications, [3-4]. The core concept lies in the custom design of MCFs to act as tunable sampled true time delay lines (TTDLs), which are the basis of many MWP signal processing functionalities, such as microwave signal filtering, radio beam-steering for phased array antennas, multi-cavity optoelectronic oscillation and arbitrary signal generation, [5].

A TTDL built upon a heterogeneous MCF implies that each core $n$ features an independent group delay, [3]. Then, at the fiber output, the signal coming from each core creates a different time delayed version of the input signal, characterized by a constant time delay difference between adjacent samples, $\Delta \tau$. In addition, if $\Delta \tau$ changes linearly with the optical wavelength, the differential delay of the TTDL can be tuned by changing the operation wavelength of the optical source. The group delay of a given core $n, \tau_{n}(\lambda)$, can be expanded in $1^{\text {st }}$-order Taylor series around an anchor wavelength $\lambda_{0}$ as $\tau_{n}(\lambda)=\tau_{n}\left(\lambda_{0}\right)+D_{n}\left(\lambda-\lambda_{0}\right)$, where $D_{n}$ is the chromatic dispersion of core $n$ at $\lambda_{0}$. For proper tunable TTDL operation, we showed in [4] that all cores must experience the same differential group delay and linearly incremental chromatic dispersion behavior at an anchor wavelength $\lambda_{0}$.

In this paper, we report, for the first time to our knowledge, the experimental demonstration of an optical beamforming network for phased array antennas built upon a 5-km dispersion-engineered heterogeneous 7-core fiber. Each core is fabricated to have a different refractive index profile to properly fulfil the requirements for sampled tunable TTDL operation while providing low intercore crosstalk.

\section{DESIGN AND FABRICATION OF A 7-CORE HETEROGENEOUS MCF AS TTDL}

We designed a heterogeneous MCF to behave as a sampled TTDL. The fiber consists of 7 trench assisted cores placed in hexagonal disposition with a $40-\mu \mathrm{m}$ core pitch and a $150-\mu \mathrm{m}$ fiber cladding. Each core was dispersion-engineered to act as an independent transmission channel with a particular group delay behavior. The core refractive index profiles comprise a $\mathrm{GeO}_{2}-$ doped core (core radius $a_{1}$ and core-to-cladding relative index difference $\Delta_{1}$ ) surrounded by a pure silica inner cladding (core-totrench distance $a_{2}$ ) and a Fluorine-doped trench (trench width $a_{3}$ and cladding-to-trench relative index difference $\Delta_{2}=0.321 \%$ ).

The design of the MCF was made using the numerical software Fimmwave from Photon Design. The fiber core parameters were properly chosen to fulfil the TTDL requirements of common group delay and linearly incremental chromatic dispersion values at the anchor wavelength, [4]. The crosstalk robustness was managed by designing neighboring cores as different as possible in terms of effective index, [6]. By properly tailoring the design parameters $a_{1}, a_{2}, a_{3}$ and $\Delta_{1}$, we set a common group delay to all cores at an anchor wavelength $\lambda_{0}=1530 \mathrm{~nm}$ and a range of chromatic dispersion $D_{n}$ values from $14.3 \mathrm{up}$ to 20.3 $\mathrm{ps} / \mathrm{km} / \mathrm{nm}$ with a $1-\mathrm{ps} / \mathrm{km} / \mathrm{nm}$ incremental dispersion at $\lambda_{0}$, respectively for cores 1 up to 7 . The minimum effective index difference between adjacent cores resulted in $7 \cdot 10^{-4}$, leading to a threshold bending radius of $80 \mathrm{~mm}$ (i.e., the minimum bending radius that ensures no phase matching between any pair of adjacent cores, [6]). We designed the cores to minimize the fiber fabrication induced errors on the TTDL performance. In general, the lower the core radius, the larger the effect on the dispersion and the group delay deviation due to fabrication errors. We thus designed the smallest cores to have the lowest chromatic dispersion values, moving the most probable mismatched cores to the outermost samples. Table 1 gathers the fiber core design parameters. 
TABLE I. CORE DESIGN PARAMETERS OF THE MCF-BASED TTDL

\begin{tabular}{c|ccccccc} 
& Core 1 & Core 2 & Core 3 & Core 4 & Core 5 & Core 6 & Core 7 \\
\hline $\boldsymbol{a}_{\mathbf{1}}(\boldsymbol{\mu} \mathbf{m})$ & 3.3 & 3.2 & 3.5 & 3.7 & 4.8 & 5.0 & 5.3 \\
$\boldsymbol{a}_{\mathbf{2}}(\boldsymbol{\mu \mathbf { m } )}$ & 5.8 & 2.4 & 4.5 & 3.7 & 5.8 & 4.6 & 3.3 \\
$\boldsymbol{a}_{\mathbf{3}}(\boldsymbol{\mu \mathbf { m } )}$ & 3.2 & 4.1 & 4.0 & 3.6 & 3.0 & 6.0 & 6.0 \\
$\boldsymbol{\Delta}_{\mathbf{1}}(\mathbf{\%})$ & 0.335 & 0.300 & 0.315 & 0.301 & 0.293 & 0.287 & 0.279
\end{tabular}

The fabrication of the MCF was carried out by YOFC company. The fabricated fiber length was $5 \mathrm{~km}$, with a couple of fanin/fan-out devices spliced at both fiber ends to inject/extract the light to/from the cores. Fig. 1(a) shows the scanning electron microscope (SEM) image of the MCF. The fiber cores can be distinguished by their different dimensions according to Table 1 parameters. Fig. 1(b) illustrates the SEM image of the $6^{\text {th }}$ core. We see that the measured dimensions of this core are in accordance with those from table 1 , but they slightly differ due to fabrication and measurement tolerances.

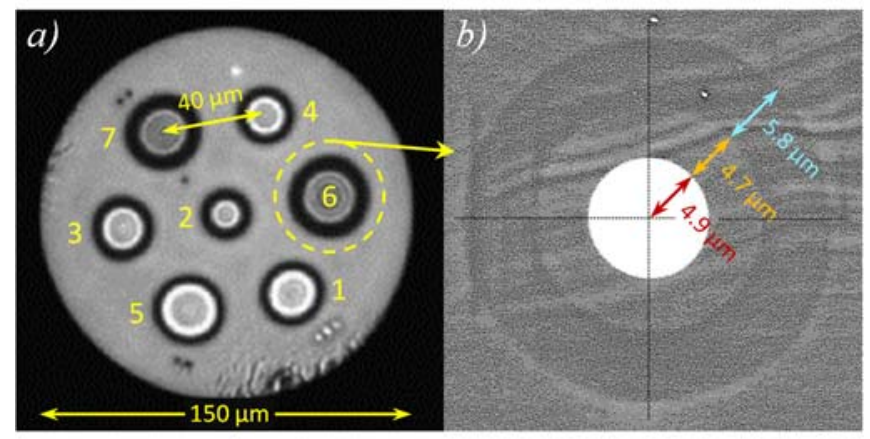

Fig. 1. (a) SEM image of the fabricated heterogeneous MCF; (b) SEM image of the fabricated $6^{\text {th }}$ core.

\section{EXPERIMENTAL CHARACTERIZATION}

The experimental characterization of the heterogeneous MCF includes losses, intercore crosstalk as wells as the chromatic dispersion and group delay behavior with the optical wavelength for each core. First, we measured the chromatic dispersion of each core at the anchor wavelength $\lambda_{0}$ by evaluating the carrier suppression effect (CSE) that affects the RF response of the 5$\mathrm{km}$ link, [7]. The inset of Fig. 2 depicts the received RF power as a function of the modulation frequency for each core at the wavelength of $\lambda_{0}=1530 \mathrm{~nm}$. The estimated chromatic dispersion values are 14.4, 15.2, 16.6, 17.6, 18.6, $19.6 \mathrm{and} 20.6 \mathrm{ps} / \mathrm{km} / \mathrm{nm}$, respectively for cores 1 up to 7 . We thus see that the fabricated cores 3 up to 7 preserve the $1-\mathrm{ps} / \mathrm{km} / \mathrm{nm}$ incremental dispersion values, while cores 1 and 2 diverge slightly from the theoretical values.

The characterization of the TTDL performance requires the evaluation of the core differential group delays behavior with the optical wavelength. We measured the differential group delays between cores via an optical interferometric based technique, [8]. First, we compensated slight mismatches between the group delays at the anchor wavelength $\lambda_{0}$ with variable optical delay lines (VDLs). These mismatches might have been caused by small discrepancies between either the design and the fabricated fiber parameters, or the different path lengths of the fan-in/fan-out devices.

Then, we measured the differential group delays between cores for different optical wavelengths ranging from 1535 up to $1560 \mathrm{~nm}$. Fig. 2 shows the measured differential group delays between core 7 and the rest of cores. Circle markers represent the experimental values while solid lines correspond to the computed differential group delays for the designed MCF. We see that the measured differential group delays of cores 3 to 7 match mostly the designed values up to a 30-nm wavelength range. As the core dispersion measure shows in the inset of Fig. 2, cores 1 and 2, which are the smallest ones, do not satisfy plenty the incremental group delay slope requirement (i.e., incremental chromatic dispersion values) since they were affected more intensely by fabrication errors. These cores can however be used to distribute 2 additional signal channels or to implement other applications that do not require space-diversity signal processing.

The fabricated MCF-based TTDL can operate in two different regimes whether we exploit the spatial or the optical wavelength diversity, what translates into different delay line possibilities within the same fiber link [3-4]. In the spatial diversity regime, the required TTDL differential delay is produced by the propagation difference experienced by two adjacent cores at a given optical wavelength. Since cores 3 up to 7 preserve similar differential group delays, we implement a 5-sample TTDL by exploiting the spatial diversity regime. The linear evolution of these 5 core differential delays within a wavelength range from 1530 up to $1560 \mathrm{~nm}$ allows us to continuously tune the basic differential delay $\Delta \tau$ of the TTDL between 0 and 150 ps. On the other hand, we can leverage all 7 cores if we operate in the wavelength diversity regime, where the differential delay comes by the propagation difference created between two adjacent wavelengths in a given core.

Finally, we measured an average intercore crosstalk at the 5-km MCF output (including the fan-in/fan-out devices) below $40 \mathrm{~dB}$ in the worst case. This level allows independent signal sample transmission. Furthermore, the average insertion losses are $5.8 \mathrm{~dB}$ per core. 


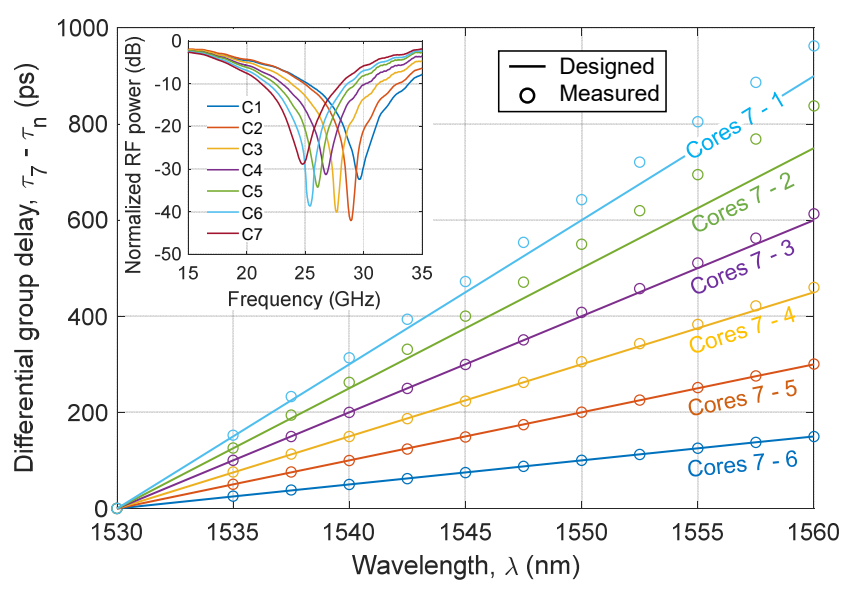

Fig. 2. Measured (circle markers) and computed (solid lines) spectral differential group delay for all the cores $\left(\tau_{\mathrm{n}}\right)$ with respect to core $\# 7$ ( $\left.\tau_{7}\right)$. Inset: Carrier suppression effect in the RF response for each core at $\lambda=1530 \mathrm{~nm}$.

\section{APPLICATION TO OPTICAL BEAMFORMING FOR PHASED ARRAY ANTENNAS}

We have experimentally demonstrated the performance of the fabricated MCF-based TTDL when it is applied to optical beamforming for phased array antennas. Optical beamforming networks follow discrete-time signal processing schemes, similarly to MWP signal filtering, with the particularity that the $N$ delayed samples are converted to the electrical domain by means of $N$ independent photodiodes. Each one of the $N$ RF output signals feeds then one of the $N$ radiating elements that comprise the phased array antenna. In the case of $1 \mathrm{D}$ architectures, the normalized angular far-field pattern of the radiated electric field, or antenna array factor $A F(\theta)$, is given by the angular Fourier transform of the overall spatial electric field distribution at the output of the radiation elements [9]:

$$
A F(\theta)=\frac{1}{N} \sum_{n=0}^{N-1} a_{n} e^{-j 2 \pi f_{R F} n(\Delta \tau-d \sin (\theta) / c)},
$$

where $\theta$ is the far field angular coordinate, $N$ is the number of samples, $a_{n}$ is the complex coefficient of sample $n, \Delta \tau$ is the differential group delay between adjacent samples, $f_{R F}$ is the RF frequency of the modulated signal, $d$ is the spacing between radiating elements and $c$ is the light speed in vacuum. From (1), we see that the angle for maximum radiating energy $\theta_{0}$ occurs when $\Delta \tau=d \sin \left(\theta_{0}\right) / c$. This beam-pointing angle $\theta_{0}$ can then be tuned by changing the differential group delay between adjacent samples, in other words, the RF phase difference between adjacent cores. It is important to notice that the use of true time delay lines for phased array antenna tunability avoids the undesired beam squint effect that arises if phase shifters are used instead, [10].

Fig. 3 shows the experimental setup to measure the phase difference between the photodetected RF signals provided by the different cores. This phase can directly be inserted in (1) instead of the phase term $2 \pi f_{R F} \Delta \tau_{7, n}$ to obtain the array factor, being $\Delta \tau_{7, n}$ the differential delay between cores 7 and $n$. As depicted in Fig. 3, the optical signal coming from a tunable laser is modulated by an electro-optical modulator (EOM) with the RF signal generated by the Vector Signal Generator (VSG). The signal is then amplified by an erbium-doped fiber amplifier (EDFA) and split and injected into all cores through the pertinent fan-in device. After 5-km MCF propagation, the signals are detected independently using seven different photodetectors (PD). Variable optical attenuators (VOAs) are used to finely equalize the output optical power of the samples for uniform amplitude distribution. VDLs were used to compensate the core group delay fabrication mismatches at the anchor wavelength or any other differential delay that is not caused by the TTDL itself. After detection, the digital phosphor oscilloscope (DPO) measures the selected RF signal. In this case, the RF signal corresponding to core 7 is taken as a reference, so we measure the phase difference (related directly to the time delay difference $\Delta \tau$ ) of the remaining cores with respect to core 7 .

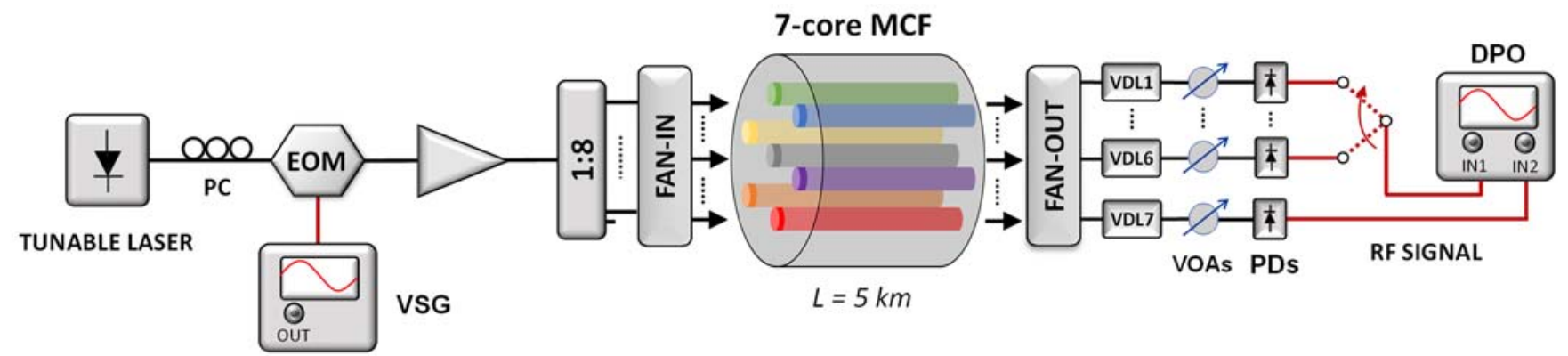

Fig. 3. Experimental setup of the optical beamforming network implemented with the fabricated heterogeneous MCF 
Fig. 4 gathers the measured electrical temporal waveforms corresponding to each core, where the RF phase difference (or time delay difference $\Delta \tau$ ) can be measured as the difference between the phase of each core with respect to that of core 7 . Table II shows the measured average RF phase difference between adjacent cores for an RF frequency of $10 \mathrm{GHz}$ and four different optical wavelengths of operation: 1540, 1545, 1550 and $1555 \mathrm{~nm}$. For proper TTDL operation, the electrical phase difference between adjacent cores must be as similar as possible (analogous to the differential delay condition). We see from Table II that, as mentioned in section 3, cores 3 up to 7 almost satisfy the differential phase (or differential group delay) condition up to the wavelength of $1555 \mathrm{~nm}$. Small discrepancies between these values and the expected ones from the differential delays measured in section 3 are attributed to the phase-measurement errors, which are actually caused by the DPO's measurement limitations.

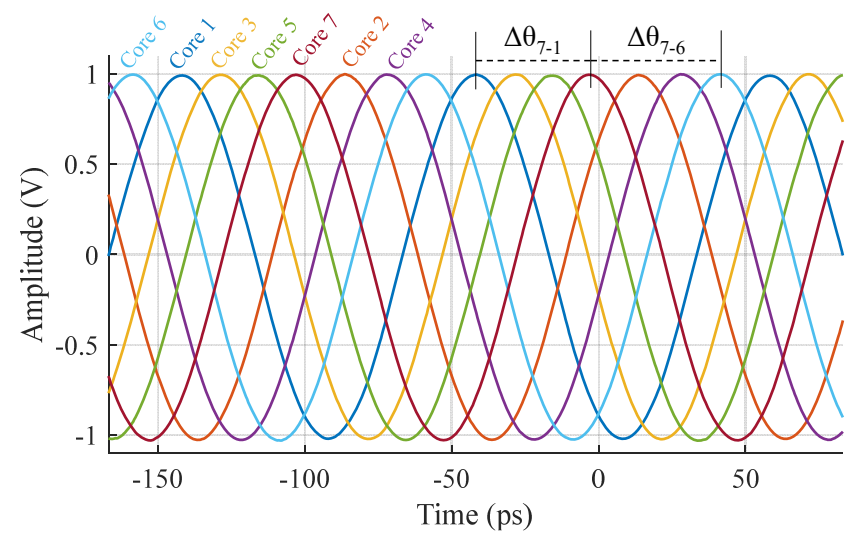

Fig. 4. Measured electrical temporal waveform at the output of each core for an RF frequency of $10 \mathrm{GHz}$ and an optical wavelength of $1540 \mathrm{~nm}$.

TABLE II. MEASURED RF PHASE DIFFERENCES BETWEEN CORES

\begin{tabular}{c|cccccc} 
Wavelength & $\begin{array}{c}\text { Cores } \\
\mathbf{1 - 2}\end{array}$ & $\begin{array}{c}\text { Cores } \\
\mathbf{2 - 3}\end{array}$ & $\begin{array}{c}\text { Cores } \\
\mathbf{3 - 4}\end{array}$ & $\begin{array}{c}\text { Cores } \\
\mathbf{4 - 5}\end{array}$ & $\begin{array}{c}\text { Cores } \\
\mathbf{5 - 6}\end{array}$ & $\begin{array}{c}\text { Cores } \\
\mathbf{6 - 7}\end{array}$ \\
\hline $\mathbf{1 5 4 0} \mathbf{~ n m}$ & $196.1^{\mathrm{o}}$ & $205.7^{\circ}$ & $199.4^{\circ}$ & $199.8^{\circ}$ & $198.9^{\circ}$ & $194.9^{\circ}$ \\
$\mathbf{1 5 4 5} \mathbf{~ n m}$ & $255.8^{\circ}$ & $328.8^{\circ}$ & $286.1^{\circ}$ & $281.3^{\circ}$ & $267.8^{\circ}$ & $270.2^{\circ}$ \\
$\mathbf{1 5 5 0} \mathbf{~ n m}$ & $321.6^{\circ}$ & $111.8^{\circ}$ & $19.2^{\circ}$ & $20.6^{\circ}$ & $15.0^{\circ}$ & $3.4^{\circ}$ \\
$\mathbf{1 5 5 5} \mathbf{~ n m}$ & $29.9^{\circ}$ & $259.6^{\circ}$ & $96.7^{\circ}$ & $90.8^{\circ}$ & $92.4^{\circ}$ & $78.6^{\circ}$
\end{tabular}

By inserting the measured values from Table II into (1), we compute the array factor created by cores 3 up to 7 . Fig. 5 represents the array factor of the 5-element phased array antenna for an RF frequency of $10 \mathrm{GHz}$ and different optical operation wavelengths. The separation between radiating elements is set to $d=1.5 \mathrm{~cm}$. The solid blue, red, yellow and purple lines represent the $A F$ for a wavelength of $1540,1545,1550$ and $1555 \mathrm{~nm}$, respectively. We see that we can easily tune the beam-pointing angle by simply changing the operation wavelength of the optical source. Here, the beam-pointing angle is swept from $-60.5^{\circ}$ at the wavelength of $1540 \mathrm{~nm}$ up to $-28.1^{\circ}$ at $1545 \mathrm{~nm}, 5.8^{\circ}$ at $1550 \mathrm{~nm}$ and $33.8^{\circ}$ at $1555 \mathrm{~nm}$. With the help of Fig. 2, one can observe that, in this situation, the maximum angle of radiation can be continuously tuned along the whole angular plane by properly changing the operation wavelength in a 20 -nm range, which corresponds to a differential group delay variation range of 100 ps.

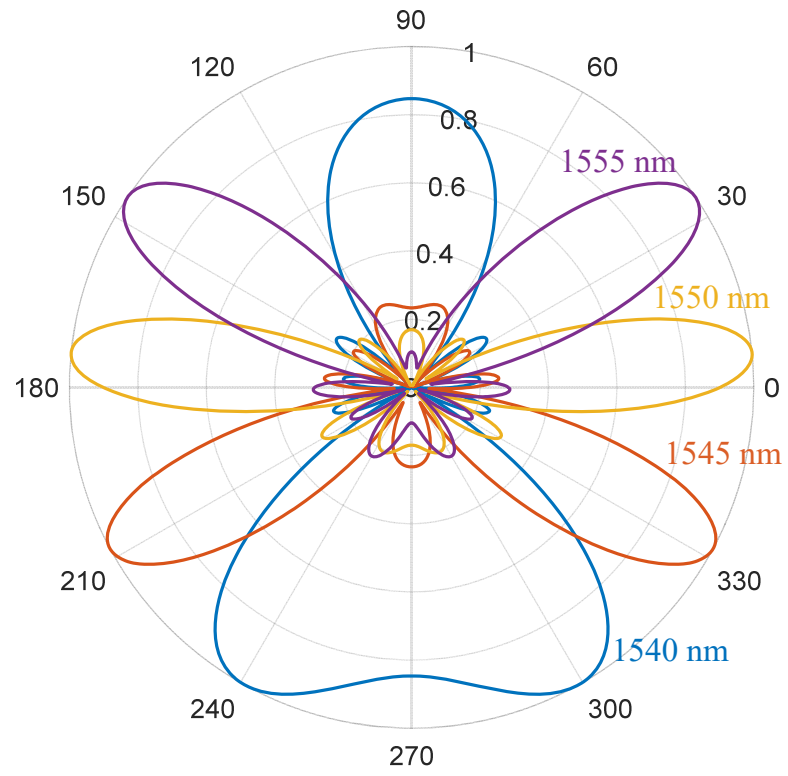

Fig. 5. Simulated antenna array factor $A F$ with the measured true time delay values for an RF frequency of $10 \mathrm{GHz}$ and different optical wavelengths. 
In addition, we demonstrated wideband operation avoiding squint-beam effects (i.e., the beam-pointing angle does not depend on the RF frequency), as expected since our optical beamforming network is built upon a TTDL. Fig. 6 represents the emulated array factor for a 5-element phased array antenna characterized by an element separation $d=1.25 \mathrm{~cm}$ for different RF frequencies. The solid blue, red, yellow and purple lines represent the $A F$ for a frequency of $8,9,10,11$ and $12 \mathrm{GHz}$, respectively. The optical wavelength of operation is $1535 \mathrm{~nm}$, which should provide around 25-ps delay difference between adjacent samples. No beamsquint degradation is observed as the beam-pointing angle is fixed at $36.5^{\circ}$ for all RF frequencies. We emulated in addition different radio beam-steering scenarios where the beam-pointing angle is modified by tuning the optical wavelength from 1540 up to $1560 \mathrm{~nm}$ while squint-beam effects are avoided for the previous radiofrequencies.

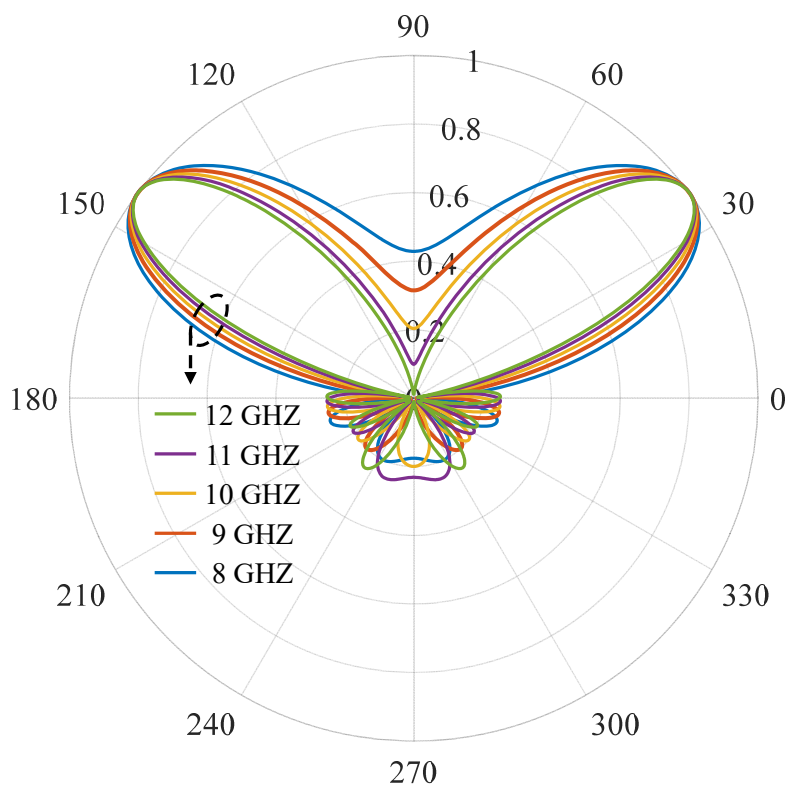

Fig. 6. Simulated antenna array factor $A F$ with the measured true time delay values for different RF frequencies and an optical wavelength of $1535 \mathrm{~nm}$.

\section{CONCLUSIONS}

The introduction of space-division multiplexing fibers into Microwave Photonics opens the door to the implementation of fiber-distributed signal processing, where both distribution and processing functionalities are provided by the same fiber medium. We experimentally demonstrated here, for the first time to our knowledge, optical beamforming networks for phase array antennas built upon a dispersion-engineered heterogeneous multicore fiber. The 5-km multicore fiber link comprises 7 different trench-assisted cores, where the refractive index profile of each core is individually addressed to fulfil the requirements for sampled tunable TTDL operation while providing low intercore crosstalk. Radio beam-steering is emulated for a 15-nm optical wavelength tuning range, demonstrating that the fabricated MCF-based TTDL satisfies the requirements for beam-squint free operation. Other RF signal processing applications, such as tunable microwave signal filtering or multicavity optoelectronic oscillation, can also be implemented by this fiber-distributed approach. Beyond Microwave Photonics, a wide range of optical or electrical signal processing systems that require to modify/compensate the chromatic dispersion or group delay (in both analogue or digital communications) could benefit from this compact and versatile fiber-distributed technique.

\section{ACKNOWLEDGEMENTS}

This research was supported by the ERC Consolidator Grant 724663, Spanish MINECO Project TEC2016-80150-R, Spanish MINECO scholarship BES-2015-073359 for S. García and Spanish MINECO fellowship RYC-2014-16247 for I. Gasulla.

\section{REFERENCES}

[1] D. J. Richardson, J. M. Fini and L. E. Nelson, "Space-division multiplexing in optical fibres,” Nat. Photonics, vol. 7, pp. $354-362$, 2013.

[2] R. Waterhouse and D. Novak, "Realizing 5G: Microwave Photonics for 5G mobile wireless systems," IEEE Microw. Magazine, vol. 16, pp. 84-92, 2015.

[3] I. Gasulla and J. Capmany, "Microwave photonics applications of multicore fibers," Photonics J., vol. 4, no. 3, pp. 877-888, 2012.

[4] S. García and I. Gasulla, "Dispersion-engineered multicore fibers for distributed radiofrequency signal processing," Opt. Express, vol. 24, pp. 20641$20654,2016$.

[5] J. Capmany, J. Mora, I. Gasulla, J. Sancho, J. Lloret and S. Sales, “Microwave photonic signal processing,” IEEE J. Lightw. Technol., vol. 31, no. 4, pp. 571-586, 2013

[6] T. Hayashi, T. Taru, O. Shimakawa, T. Sasaki and E. Sasaoka, "Design and fabrication of ultra-low crosstalk and low-loss multi-core fiber," Opt. Express, vol. 19, pp. 16576-16592, 2011.

[7] G. H. Smith, D. Novak and Z. Ahmed, "Novel technique for generation of optical SSB with carrier using a single MZM to overcome fiber chromatic dispersion," in International Topical Meeting on Microwave Photonics. MWP '96 Technical Digest. Satellite Works, Kyoto, Japan, 1996. 
[8] C. Dorrer, N. Belabas, J. Linkforman and M. Joffre, "Spectral resolution and sampling issues in Fourier-transform spectral interferometry," J. Opt. Soc. Am. B, vol. 17, no. 10, pp. 1795-1802, 2000.

[9] R. J. Mailloux, Phased Array Antenna Handbook. 2nd edition., Dedham, MA: Artech House Publishing Co., 2000.

[10] I. Frigyes and A. Seeds, "Optically generated true-time delay in phased-array antennas," IEEE Transactions on Microwave Theory and Techniques, vol. 43, pp. 2378-2386, 1995 